\title{
Reproductive Care of Childhood and Adolescent Cancer Survivors: A Twelve-Year Evaluation
}

\author{
Antoinette Anazodo ${ }^{1}$, Sumin Choi ${ }^{2}$, Christina Signorelli ${ }^{3}$, Sarah Ellis ${ }^{3}$, Karen Johnston ${ }^{1}$, \\ Claire Wakefield ${ }^{4}$, Rebecca Deans ${ }^{1}$, Kristen Neville $^{5}$, and R. J. Cohn ${ }^{1}$ \\ ${ }^{1}$ Sydney Children's Hospital Randwick \\ ${ }^{2}$ University of New South Wales \\ ${ }^{3}$ Sydney Children's Hospital \\ ${ }^{4}$ University of NSW \\ ${ }^{5}$ University of New South Wales Faculty of Medicine
}

June 8, 2020

\begin{abstract}
Background: Reproductive complications for cancer survivors are identified as one of the top unmet needs. Current models of care do not routinely incorporate reproductive follow-up for cancer patients. The Kids Cancer Centre have had a one stop survivorship clinic which includes a gynecologist and fertility specialist. Methodology: To inform the future development of our reproductive survivorship care we audited this service over a twelve-year period reviewing who used the service and their gonadotoxic risk, their reproductive needs and concerns. Main results: 278 patients were seen (397 consultations), including 189 female patients (68.0\%). Median age at follow up was 25.0 years (range=6-50) and they were 19.2 years from their primary diagnosis (range $=3-46$ ). We identified 10 themes of reproductive need. Patients had on average 2.5 reproductive concerns documented per consultation (range 1-5). The three most commonly documented concerns at initial consultation related to fertility status (43.9\%), endocrine dysfunction (35.3\%), and contraception advice (32.4\%). In patients younger than 25 years discussions were predominately about endocrine dysfunction, fertility status and contraception, while dominant themes for 26-35 years olds were fertility status, reproductive-related health prevention strategies, contraception and endocrine dysfunction. Survivors aged 36-45 prioritised fertility status, pregnancy, and contraception. Fertility preservation ( $\mathrm{p}=0.05)$, preventative health strategies $(\mathrm{p}=0.001)$, and contraception advice $(\mathrm{p}<0.001)$ were more commonly discussed by females than males. Conclusion: Longitudinal reproductive follow up care is important, as patients have a number of ongoing reproductive concerns which change over time. Our data can assist in informing the model of care.
\end{abstract}

\section{Hosted file}

Reproductive Survivorship Paper 6th June 2020.doc available at https://authorea.com/users/ 330978/articles/457731-reproductive-care-of-childhood-and-adolescent-cancer-survivors-atwelve-year-evaluation

\section{Hosted file}

Table 1 - Themes derived from reproductive health consultations.docx available
https://authorea.com/users/330978/articles/457731-reproductive-care-of-childhood-and-
adolescent-cancer-survivors-a-twelve-year-evaluation

\section{Hosted file}

Table 2- Patient demographic and clinical characteristics.docx available at https://authorea. 
com/users/330978/articles/457731-reproductive-care-of-childhood-and-adolescent-cancersurvivors-a-twelve-year-evaluation

\section{Hosted file}

Table 3 - Consultation Characteristics Over Time.docx available at https://authorea.com/ users/330978/articles/457731-reproductive-care-of-childhood-and-adolescent-cancersurvivors-a-twelve-year-evaluation

\section{Hosted file}

Figure 1 - Top five reproductive topics discussed by age group.docx available at https:// authorea.com/users/330978/articles/457731-reproductive-care-of-childhood-and-adolescentcancer-survivors-a-twelve-year-evaluation

\section{Hosted file}

Figure 2- Trends in reproductive topics raised over time by year of consultation.docx available at https://authorea.com/users/330978/articles/457731-reproductive-care-ofchildhood-and-adolescent-cancer-survivors-a-twelve-year-evaluation 